\title{
Subjective and objective neuropsychological
}

\section{abnormalities in a psychosis prodrome clinic*}

\author{
MARTIN HAMBRECHT, MICHAEL LAMMERTINK, JOACHIM KLOSTERKÖTTER, \\ EVELINE MATUSCHEK and RALF PUKROP
}

\author{
Background Predicting transitions to \\ psychosis is difficult. Neuropsychology \\ might facilitate predictions.
}

\begin{abstract}
Aims To report preliminary data on self-perceived and objectively measurable neurocognition in prodromal patients of the first German early recognition centre.

Method Subjective neuropsychological disturbances were assessed in 51 patients with potentially prodromal symptoms of schizophrenia. Initial neurocognitive functioning was compared with matched normals and patients with schizophrenia.
\end{abstract}

Results Self-perceived deficits mostly concerned perception, cognition and stress reactivity. Five transitions happened during the 15-month follow-up. Recently emerging or intensifying deficits were to some extent predictive of transition. Persons at risk performed worse than controls on objectively measured verbal capacity, attention and memory functions.

\section{Conclusions Neuropsychological deficits, either self-perceived or objectively measured, characterise persons at risk for schizophrenia and may contribute to predicting transitions.}

\section{Declaration of interest Funded by the Cologne Faculty of Medicine (Köln Fortune) and Federal Ministry for Education and Research BMBF, German Schizophrenia Research Network (grant 0I GI 993x).}

* Presented in part at the European First Episode Schizophrenia Network Meeting,Whistler BC, Canada, 28 April 200I.
Several studies have revealed that psychotic symptoms on average lasting 1 year and non-psychotic prodromal symptoms lasting several years occur prior to diagnosis and treatment of schizophrenia (e.g. Häfner et al, 1993). The duration of untreated illness in early psychosis has been found to be associated with course and outcome (e.g., Huber et al, 1979; Loebel et al, 1992; McGlashan, 1999). The consequences of untreated illness and particularly untreated psychosis are severe on all levels of psychological and social functioning. A large representative first-episode study (Häfner et al, 1995, 1999) found that during the prodromal stage $80 \%$ of patients have depression and that social decline and stagnation in personal development already start before the first admission. Reducing the duration of untreated psychosis (DUP) and/or the duration of untreated illness (DUI) has become a major focus of early detection and early recognition activities worldwide, including the Personal Assessment and Crisis Evaluation (PACE) Clinic in Melbourne (McGorry et al, 1996), the Tydlig Intervention vel Psychoser (TIPS) project in Stavanger, Norway (Larsen et al, 1998), the PRIME Research Clinic at Yale University and the Recognition and Prevention Program (RAP) on Long Island (Cornblatt et al, 1998).

\section{Indicated prevention, risk and neuropsychological deficits}

The combination of a long duration of untreated symptoms and their severe consequences calls for early intervention in the sense of 'indicated prevention' (Mrazek \& Haggerty, 1994). Indicated prevention, however, requires prediction and prediction research encounters problems. DSM-III-R prodromal symptoms, for instance, have been found to be unspecific (American Psychiatric Association, 1987). Using odd speech, odd behaviours, social withdrawal, or other DSM-III-R prodromal symptoms would result in $8-30 \%$ false positive and $7-23 \%$ false negative diagnostic classifications (Jackson et al, 1995). Even a decision rule developed by McGorry et al (2000) with sophisticated statistical methods and based on a combination of retrospectively assessed prodromal variables, pre-psychotic deterioration, premorbid functioning, and prodromal duration performed only equivalently to the single variable 'duration of prodrome'.

Yung et al (1998) employed the more simple definition of 'at-risk mental states': either brief limited intermittent psychotic symptoms, or attenuated psychotic symptoms, or a genetic/obstetric risk plus decline in functioning. These states were reported to lead to psychosis in $40 \%$ of the cases. However, these are patients who already have psychotic symptoms - although limited in time or severity - and seem to be already on the edge of psychosis, and prediction might be considered as less informative. In individual cases, a positive family history is extremely helpful for prediction but only a minority of patients have a genetic risk and the same is true for obstetric complications. Brain imaging, in particular functional magnetic resonance imaging (MRI), is promising for the future but better methodology is needed, and presently no definite brain imaging marker for schizophrenia has been established (Lawrie \& Abukmeil, 1998).

From the point of view that schizophrenia is a disorder of informationprocessing, it makes sense to add neuropsychological abnormalities to these indicators in order to calculate a 'risk profile'. Within a multi-level assessment of potential risk indicators for schizophrenia, both objective and subjective, i.e. self-perceived, neuropsychological deficits may contribute information for prediction. A large retrospective study (Häfner et al, 1992) found that impairments of cognition and concentration are among the first signs that patients later diagnosed with schizophrenia had perceived initially. A prospective study of patients referred to university departments because of diagnostic problems demonstrated that subtle self-perceived cognitive and perceptual aberrations can predict the transition to schizophrenia over a long-term follow-up (Klosterkötter et al, 2001). During the mean follow-up period of 9.6 years, 79 out of 160 initially assessed patients had a transition to schizophrenia. The absence of specific self-perceived 
prodromal symptoms excluded subsequent schizophrenia with a probability of $96 \%$ (sensitivity 0.98 ; false-negative predictions $1.3 \%$ ), whereas their presence predicted a transition with a probability of $70 \%$ (specificity 0.59 ; false-positive predictions $20 \%$ ). Certain disturbances, such as thought interference, thought blockages, pressure of thought, visual distortions, or disturbed reception of language, predicted schizophrenia with a probability up to 91\% (specificity 0.85-0.91; false-positive predictions $1.9 \%-7.5 \%)$. Thus, the assessment of self-perceived neuropsychological disturbances, for which Gerd Huber has coined the term 'basic symptoms' (Gross, 1989), is particularly helpful to rule out later schizophrenia; patients who did not have such complaints were very unlikely to encounter a transition to psychosis within the following decade.

Furthermore, a wide range of objectively measured neuropsychological deficits is well documented among actively psychotic or remitted, and first-episode or chronic patients with schizophrenia, their first-degree relatives, children at heightened risk for schizophrenia and individuals with schizotypic or schizophrenia-spectrum disorders or hypothetically prone to psychosis. Thus, information-processing abnormalities could represent a robust bio-behavioural liability marker for schizophrenia that could play an important role in identifying prodromal states and predicting the probability of transition to psychosis. Several studies have described global neurocognitive performance deficits (e.g. Mohamed et al, 1999), others have shown selective deficits over and above general impairment emphasising (verbal) memory or executive functions (e.g. Saykin et al, 1994; Hutton et al, 1998). Therefore, at this early stage of research we decided to use a wide variety of tests potentially relevant for patients at risk for schizophrenia, including various stages of information-processing, such as perception, attention, working memory, verbal and visual long-term memory systems, verbal capacities and flexibility in problem-solving.

On the basis of these considerations, we sought to investigate the role of subjective and objective neuropsychological deficits in prodromal patients. The following questions were addressed.

(a) Which self-perceived neuropsychological disturbances (basic symptoms) are characteristic for a prodromal sample seen by a new early detection centre at the initial assessment?

(b) How severe is psychopathology (positive/negative symptoms, schizotypal traits) in these prodromal patients?

(c) Does this sample show objective neurocognitive performance deficits at the initial assessment in comparison with demographically matched healthy controls and patients with schizophrenia?

(d) Did those prodromal patients who later developed schizophrenia during a 15month follow-up differ initially from those who did not with regard to selfexperienced deficits and observed psychopathology?

Because of the small number of expected transitions to psychosis within the short follow-up interval, the anticipated results were conceived as preliminary but helpful for refining hypotheses for a more comprehensive longitudinal study interval.

\section{METHOD}

\section{The Cologne early recognition centre}

The first German early recognition centre FETZ (FrühErkennungs- und TherapieZentrum für Psychische Krisen) was initiated in Cologne in 1997 as a specialised outpatient service (prodrome clinic) to identify and treat persons at risk of schizophrenia. The primary focus is not on reducing DUP but on detecting persons before the onset of psychotic symptoms in order to reduce DUI at an earlier stage. FETZ is located on the campus of the University Hospital of Cologne - close to, but separate from, the Department of Psychiatry and Psychotherapy, with access to its neuropsychology and neurophysiology laboratories. On the basis of a continuous information campaign for professionals in the whole medico-social field, FETZ established a local early recognition network with periodical newsletters and seminars. From time to time, there were newspaper articles, radio and TV interviews and a special 'early recognition week' to address the general public. A homepage (www.fetz. org) provides information and facilitates contact (100 visitors weekly). Roughly half of the referrals now come from psychiatrists and psychotherapists, a quarter through relatives and the rest from various sources including counselling centres, school psychologists and self-referrals.

\section{Initial assessment}

After the pilot phase of introducing FETZ, establishing pathways, testing and implementing diagnostic and therapeutic tools, the centre has provided systematic assessment since October 1998. Standardised treatment was implemented in January 2000 through multi-centre psychological and pharmacological intervention trials within the German Research Network on Schizophrenia. This paper is based on the initial 10 months in which FETZ offered standard individual clinical treatment. Within this period, FETZ received about 250 telephone inquiries from (potential) patients or relatives and more than 120 inquiries from professionals. After the basic screening by telephone 158 persons were invited to a personal interview; 135 attended and were screened for the inclusion criteria, i.e., at least two of the nine symptoms below that had emerged recently:

(a) marked social withdrawal or isolation;

(b) marked impairment in role as wageearner, student or home-maker;

(c) odd beliefs or magical thinking, influencing behaviour and inconsistent with cultural norms;

(d) unstable ideas of reference ('subjectcentrism');

(e) interference of thoughts;

(f) pressure or perseveration of thoughts;

(g) peculiar perceptual disturbances without organic cause;

(h) impaired capacity to make contact despite a wish to make contact;

(i) increased emotional reactivity in response to everyday events.

These items represent a combination of the most specific DSM-III-R prodromal symptoms $(\mathrm{a}-\mathrm{c})$ according to Jackson et al (1995) and of predictive self-perceived disturbances (d-i), i.e. 'basic symptoms' (Klosterkötter et al, 2001). The latter were assessed with the Bonn Scale for the Assessment of Basic Symptoms (BSABS; Gross et al, 1987). This instrument gives operational definitions of subtle, selfperceived deviations of cognition, language, perception, mood, energy and tolerance to normal stress, along with typical statements of patients and examples of questions for a structured interview. Specific, as well as general, guiding questions for symptom categories are given. On the basis of the patient's description of a complaint, the 
interviewer decides whether the symptom in question is rated as 'present', 'questionably present' or 'absent'. For the present study, a shortened 66-item version of the BSABS was used.

\section{Sample characteristics}

Persons with established schizophrenia, organic brain syndromes, dependency on alcohol or drugs and refusers were excluded from the project and referred to other specialised services. A total of 71 patients were thus included, of which 51 could be followed up on average 15.1 months later (9 persons refused to participate in the follow-up and 11 could not be contacted). The age range of the follow-up sample was 15-39 years, the mean age was 25.5 years (s.d. 6 years) and 42 patients were male $(82 \%)$. Initially about half of the patients had been referred from psychiatrists or psychotherapists in independent practice asking for specialised diagnostic assessment, a third came as self-referrals or were brought in by family members after reports on FETZ in the media and the remainder were referred from various psychosocial services, e.g. school social workers.

Because neuropsychological testing was not available in the first months of FETZ, only a subsample of 29 prodromal patients (mean age 23.10 years, s.d. 4.43, range 1531 years; $75.9 \%(n=22)$ male; 11.81 years of education; all never medicated by neuroleptics) selected by the above-defined criteria was investigated by a neuropsychological test battery. These prodromal patients were compared with 29 patients with schizophrenia (mean age 22.97 years, s.d. 3.81, range $17-32$ years; $75.9 \%(n=22)$ male; 11.14 years of education) and 29 healthy controls (mean age 23.97 years, s.d. 3.04, range $17-31$ years; $75.9 \%(n=22)$ male; 12.38 years of education). Patients with schizophrenia were diagnosed according to DSM-IV criteria for schizophrenia using the structured clinical interview for DSMIV, axis-I (SCID-I; Wittchen \& Fydrich, 1997). Those with concomitant axis-I diagnoses, history of neurologic disorders and present substance misuse were excluded. Patients with schizophrenia had a median number of admissions to hospital of 1 (range $0-5$ ), and a mean age range at onset of the illness of 21.68 years (s.d. 3.80). Severity of illness measured by the Positive and Negative Syndrome Scale (PANSS; Kay, 1991) was 20.57 (s.d. 8.66) for negative symptoms, and 16.21 (s.d. 5.51) for positive symptoms. Seventeen patients with schizophrenia received atypical neuroleptics, five were treated by typical neuroleptics and seven were unmedicated for at least 4 weeks prior to the investigation.

Prodromal patients did not differ from healthy controls and patients with schizophrenia in age $(F=0.588 ; P=0.558)$, gender $\left(\chi^{2}=0.00 ; P=1.00\right)$ and educational level, although healthy controls had a higher level of education than patients with schizophrenia $(F=4.53 ; P=0.014)$. However, all three groups differed significantly in premorbid verbal IQ scores $(F=24.55$; $P<0.01$ ), with normal controls showing the highest and patients with schizophrenia the lowest level. Verbal IQ was measured by the Mehrfachwahl-Wortschalz-Intelligenztes (Lehrl, 1995), which consists of 37 series of 4 non-words and 1 word that has to be identified.

\section{Neuropsychological assessment Perception (visual backward masking)}

Target stimuli (letters F, H or T) were presented for $12 \mathrm{~ms}$ in the centre of a computer screen followed by 1 of 4 possible masking conditions: noise (random dot pattern) or letter pattern masking stimulus after short ( $42 \mathrm{~ms})$ or long $(104 \mathrm{~ms})$ interstimulus intervals. The session consisted of 3 blocks of 30 trials each including 6 trials of each masking condition and 6 no-mask control trials presented in a pseudo-random order. The score for the present study was the average number of hits over all 3 blocks and 4 masking conditions.

\section{Attention (Continuous Performance Test, identical pairs version; Cornblatt et al, 1989)}

The number of hits averaged over 300 trials (nonsense shape targets and four-digit numbers) was calculated.

\section{Spatial working memory delayed response task}

The stimulus material was arranged as described in the literature (Spitzer, 1993) and consisted of 16 black dots displayed on the white screen of a touch-sensitive monitor $(1200 \times 1600$ pixels $)$. During each trial the screen was blank for $3000 \mathrm{~ms}$ and then the subject observed a central fixation cross for $700 \mathrm{~ms}$; a black dot then appeared in 1 of 16 possible positions $\left(360 / 16^{\circ}\right.$ distance) of a circular arrangement for $200 \mathrm{~ms}$. During the subsequent delay period of $15 \mathrm{~s}$ the subjects saw a three-digit number from which they had to count backwards in threes to avoid active maintenance of the target stimulus. After the number had disappeared they had to indicate with the index finger of the dominant hand the position of the dot presented before the distractor task. The session consisted of 16 trials and the average Euclidean distance in pixel coordinates of responses from the correct position of the dot was calculated.

Verbal memory and learning (Auditory Verbal Learning Test; Lezak, 1983)

A free recall (average number of words in trials 1-5) and a recognition score (after a 30 minute delay period) were documented.

\section{Verbal fluency}

The average number of words generated in 1 minute in response to three letters and one category was used as an indicator of word fluency.

\section{Visual memory (Rey-Osterrieth Complex Figure Test; Rey, 1964)}

A loss score was calculated as the difference between copy performance and delayed recall performance.

\section{Abstraction/Flexibility (Wisconsin Card Sorting Test; Heaton et al, 1993)}

The percentage of perseverative errors was used as the central parameter.

\section{Statistical analyses}

Frequency counts and descriptive statistics were calculated for initial basic symptoms, observed and self-perceived psychopathology. Differences in initial psychopathology between those patients at risk who experienced a transition to psychosis during follow-up and those who did not were tested by Mann-Whitney U-tests. Fisher's exact test statistics, sensitivity, specificity and related measures were calculated in the prospective part. Data on neuropsychology were analysed by non-parametric statistics because of small sample size and asymmetrical distributions of raw data. Mann-Whitney $U$-tests were used to analyse the difference between prodromal patients and the two control groups (normal controls and patients with schizophrenia). To estimate the impact of verbal IQ on neuropsychological test data, Spearman 
correlation coefficients were calculated within each group.

\section{RESULTS}

\section{Self-perceived deficits and psychopathology at inclusion}

Self-perceived neuropsychological disturbances were assessed with the BSABS (Gross et al, 1987). Most complaints concerned perception, cognition and stress reactivity (Table 1). Patients were always asked about the course of the symptom over time, i.e. 'Did it emerge recently or has it definitely increased in severity?'

Observed psychopathology was rated with the Positive and Negative Symptom Scale (PANSS; Kay, 1991). Although the general level of positive and negative symptomatology was relatively low (Table 2), paranoid ideation, agitation, affective flattening, emotional withdrawal and apathy were present in more than half of the sample. Self-ratings for the schizotypal traits of perceptual aberration, physical anhedonia and magical ideation (Chapman et al, 1976; Scherbarth-Roschmann \& Hautzinger, 1991) showed a moderate prevalence of these traits (Table 2).

\section{Follow-up and prediction}

At follow-up, 41 of 51 patients $(80.4 \%)$ reported some kind of therapy during the follow-up period; one-third inside and two-thirds outside of FETZ. Fourteen patients $(27.5 \%)$ reported psychotherapy, $12(23.5 \%)$ reported pharmacotherapy and $15(29.4 \%)$ had a combination of both. Within the follow-up period of 15 months, $5(9.8 \%)$ out of 51 patients had a transition to psychosis. As defined by F20.0 of ICD-10 (World Health Organization, 1992) these 5 patients were diagnosed with the paranoid subtype of a schizophrenic disorder according to DSM-IV (American Psychiatric Association, 1994), or with a schizophreniform disorder because of the relatively short duration of their psychotic symptoms. All five were male and their mean age was 21.9 years. The interval between initial assessment and transition ranged from 3 to 17 months (on average 8.6 months). Two patients were without therapy in this interval, two were treated with psychotherapy and with antipsychotics and one person received only antipsychotics.

Patients who had a transition to psychosis initially showed higher levels of psychopathology (both positive and negative symptoms assessed by the PANSS) and described more schizotypal traits, particularly magical ideation, than patients without transition (Table 2). Because of the small number of transitions, these differences, however, reached only the level of a statistical trend with regard to magical ideation.

A preliminary analysis of this small data-set reveals that self-perceived neuropsychological disturbances (basic symptoms) could have predictive power even for this short follow-up. A subset of basic symptoms that had been found to be highly predictive over a long-term follow-up (Klosterkötter et al, 2001) can be useful for a short-term prediction, if the criteria of recency and intensity are taken into consideration; those basic symptoms that had increased in severity according to our clinical judgement or that had emerged shortly before the initial assessment demonstrated predictive value (Table 3 ). The resulting likelihood ratio (=sensitivity/1 - specificity) was 3.33 (Jaeschke et al, 1994).

\section{Neuropsychological deficits of prodromal patients}

Normal controls showed the highest performance levels; prodromal patients scored in between and patients with schizophrenia performed worst on all neurocognitive test parameters (Table 4). Prodromal patients differed significantly from normal controls in attention $(\mathrm{Z}=-2.02 ; P=0.044)$, verbal memory-free recall $(Z=-2.01 ; P=0.044)$, verbal fluency $(Z=-3.11 ; P=0.002)$ and visual memory $(Z=-2.46 ; \quad P=0.014)$. There was no difference in visual backward masking, spatial working memory, verbal recognition memory and Wisconsin Card Sorting Test performance. However, when $\alpha$ error was strictly controlled by a Bonferroni procedure to an overall level of 0.05 , verbal fluency was the only paradigm indicating a significant difference.

Prodromal patients showed significantly better performance than patients with schizophrenia on all neuropsychological functions (Table 4). Controls differed significantly from patients with schizophrenia on $\alpha=1 \%$ for all neuropsychological functions (not shown in Table 4). There was a tendency for verbal fluency to correlate with verbal IQ $\left(r_{\mathrm{s}}=0.34 ; P=0.073\right)$ in the prodromal sample. No other neuropsychological parameters correlated significantly with verbal IQ in patients at risk of psychosis. Within the schizophrenia sample verbal IQ showed significant relationships with verbal fluency $\left(r_{s}=0.45 ; P=0.014\right)$ and Wisconsin Card Sorting Test perseverative errors $\left(r_{s}=-0.40 ; P=0.031\right)$. There were no significant relationships between verbal IQ and neurocognitive functions in normal controls.

\section{DISCUSSION}

This pilot study has methodological limitations, particularly concerning the prospective part. Twenty patients $(28 \%$ of the initial sample) could not be included in the follow-up and their course is unknown. The current research projects of FETZ, therefore, stay in contact with patients at least monthly by telephone.

\section{Feasibility of short-term predictions}

At only 15 months, the follow-up interval was very short and more transitions in this sample can be expected in the future, given the fact that Klosterkötter et al (2001) reported a mean interval between assessment and transition of 5.6 years. Inclusion criteria are extremely influential in prospective early detection and early intervention studies, but there is no consensus about which criteria should be employed because the empirical basis of prediction is still weak. This situation is mirrored in the present study, where the employed inclusion criteria were preliminary.

In spite of these limitations, the study shows that self-perceived neuropsychological deficits may add information even for relatively short-term prediction, if their timing and intensity is taken into consideration. When this information can be elicited, the likelihood ratio of 3.33 generates a small, but potentially relevant change in the probability of transition within a follow-up of only 15 months (Jaeschke et al, 1994).

From a practical point of view, the study demonstrates that a special early recognition service can quickly become an accepted part of a region's health care services and attract a considerable number of persons at risk for psychosis. It is noteworthy that in the present study, three of five patients with later transitions had received antipsychotic medication before they became psychotic. Because treatment was neither applied nor monitored in a systematic way, these limited clinical observations 
Table I Prevalence of self-perceived disturbances at the initial interview (rated as definitely present)

\begin{tabular}{|c|c|c|}
\hline BSABS item no. & Self-perceived disturbance & $\%(n=5 I)$ \\
\hline C.1.5 & Difficulties concentrating & 66.7 \\
\hline A.8.2+B.1.3 & Impaired tolerance to certain social situations of everyday life that are primarily emotionally neutral & 58.8 \\
\hline $\begin{array}{l}\text { At least one of } 15 \text { symptoms } \\
\text { (C.2.IsI to C.2.3s } 12 \text { ) }\end{array}$ & Visual perceptual disturbances' & 52.9 \\
\hline A.8.I+B.1.2 & Impaired tolerance to unusual, unexpected or specific novel demands & 49.0 \\
\hline A.6.4 & Decrease in the need for contact with others & 47.1 \\
\hline C.1.3 & Thought pressure' & 43.1 \\
\hline $\begin{array}{l}\text { At least one of four symptoms } \\
\text { (C.2.4sI to C.2.5s2) }\end{array}$ & Acoustic perceptual disturbances' & 43.1 \\
\hline A.8.3+B.1.4 & Impaired tolerance to working under pressure of time or rapidly changing different demands & 37.3 \\
\hline C.I.7 & Disturbance of expressive language & 35.3 \\
\hline A.7.I & Decrease in the ability to maintain or initiate social contacts & 35.3 \\
\hline C.2.8 & Feeling overwhelmed by stimuli, hyperdistractability & 33.3 \\
\hline C.I.I & Thought interference' & 33.3 \\
\hline A.6.I & Change in mood and emotional responsiveness & 31.4 \\
\hline B.I.I & Impaired tolerance to everyday stress or routine work & 29.4 \\
\hline C.I.4 & Thought blockages' & 27.5 \\
\hline C.I.6 & Disturbance of receptive language, either heard or read' & 27.5 \\
\hline C.3.2 & Motor blockages & 27.5 \\
\hline C.1.9 & Difficulties holding things in mind for less than half an hour (short-term memory) & 27.5 \\
\hline B.2.I & Increased emotional reactivity in response to everyday events & 27.5 \\
\hline B.2.2 & Increased emotional reactivity in response to routine social interactions & 27.5 \\
\hline A.6.2 & Decreased capacity to discriminate between different kinds of emotions & 25.5 \\
\hline C.I.13 & Lack of 'thought energy' or goal-directed thoughts & 25.5 \\
\hline A.7.2 & $\begin{array}{l}\text { Disturbances of emotional responsiveness as characterised by a decrease in facial expression, intonation and } \\
\text { communication gestures }\end{array}$ & 23.5 \\
\hline C.2.II & Derealisation' & 23.5 \\
\hline D.8 & Sensations of the body or parts of it being abnormally heavy, light, empty, falling or sinking & 23.5 \\
\hline C.I.12 & Slowed-down thinking & 23.5 \\
\hline C.I.17 & Unstable ideas of reference ${ }^{\prime}$ & 23.5 \\
\hline C.I.2 & Thought perseveration' & 21.6 \\
\hline C.I.10 & Disturbance of retrieval of presently required knowledge from long-term memory & 19.6 \\
\hline D.6 & Thermal bodily sensations, unusual coldness or warmth & 19.6 \\
\hline D.3 & Unusual bodily sensations of pain in a distinct area & 19.6 \\
\hline D. 14 & Dysaesthetic crisis (unusual bodily sensation plus central-vegetative disturbance ${ }^{2}$ or fear of dying any minute) & 19.6 \\
\hline C.I.15 & Decreased ability to discriminate between ideas and perception, fantasy and true memories' & 17.6 \\
\hline C.2.9 & Captivation of attention by details of the visual field & 17.6 \\
\hline C.3.I & Motor interference exceeding simple lack of coordination & 17.6 \\
\hline C.3.3 & Loss of automatic skills & 17.6 \\
\hline D.II & Vestibular sensations of vertigo, unsure gait, walking on moving ground & 17.6 \\
\hline C.I.8 & Difficulties holding things in mind for seconds (immediate recall) & 15.7 \\
\hline D.I & Unusual bodily sensations of numbness or stiffness & 15.7 \\
\hline B.2.3 & Increased emotional reactivity in response to strangers' misfortune & 15.7 \\
\hline C.2.6 & Disturbances of olfactory, gustatory or tactile perception & 11.8 \\
\hline D.IsI & Psychosomatic bodily depersonalisation & 11.8 \\
\hline D.7 & Bodily sensations of movement, pulling, pressure inside the body or on its surface & 11.8 \\
\hline D.5 & Electric bodily sensations, feelings of being electrified & 11.8 \\
\hline C.I.16 & Disturbance of abstract thinking ('concretism') & 9.8 \\
\hline D.2 & Bodily sensations of motor weakness, 'paralysis' & 9.8 \\
\hline D.4 & Migrating bodily sensations 'wandering' through the body & 2.0 \\
\hline
\end{tabular}

BSABS, Bonn Scale for the Assessment of Basic Symptoms.

I. Subset of highly predictive prodromal symptoms (Klosterkötter et al, 200I).

2. May include a range of 'psychosomatic' autonomic disturbances, including irregularities of heartbeat, respiratory abnormalities, sudden heavy sweating, flushing or livid discolouration of hands and face, giddiness, nausea and vomiting. 
Table 2 Observed psychopathology on Positive and Negative Symptom Scale (PANSS) and self-rated schizotypal traits at initial assessment in patients with and without transition (mean (s.d.))

\begin{tabular}{lcccc}
\hline & Transition $n=4$ & No transition $n=40$ & Total $n=44$ \\
\hline PANSS, positive score & $14.0(5.6)$ & $11.1(2.7)$ & $11.2(2.9)$ \\
PANSS, negative score & 16.0 & $(3.5)$ & $13.4(5.0)$ & $13.6(4.9)$ \\
PANSS, general score & 32.0 & $(6.2)$ & $29.8(4.2)$ & $30.0(4.4)$ \\
PANSS, total score & $62.0(11.5)$ & $54.2(8.8)$ & $54.8(9.1)$ \\
Magical ideation' & $7.75(6.4)$ & $2.93(2.7)$ & $3.36(3.4)$ \\
Physical anhedonia & $5.25(3.1)$ & $4.15(4.2)$ & $4.25(4.1)$ \\
Perceptual aberration & $15.25(7.8)$ & $13.30(8.1)$ & $13.48(8.0)$ \\
\hline
\end{tabular}

I. Mann-Whitney-test: $P<0.10$ (transition v. no transition). Missing data for 7 patients ( $I$ in the transition group, 6 in the no transition group).

Table 3 Prodromal symptoms and transition to psychosis within 15 months

\begin{tabular}{lccc}
\hline & $\begin{array}{c}\text { Selected basic symptoms' recently } \\
\text { emerged or became more intense }\end{array}$ & $\begin{array}{c}\text { No changes in these basic } \\
\text { symptoms }\end{array}$ & Total \\
\hline Transition & 4 & 1 & 5 \\
No transition & II & 35 & 46 \\
Total & 15 & 36 & 51 \\
\hline
\end{tabular}

Sensitivity $=0.80$; rate of correct classifications $=76.5 \%$. Specificity $=0.76$. Fisher's exact test: $P=0.022$.

I. The subset of highly predictive prodromal symptoms (Klosterkötter et al, 200I).

cannot be generalised. Systematic trials of early interventions (McGorry et al, 1999) suggest the preventive effects of antipsychotics and/or cognitive-behavioural therapy.

\section{Neuropsychological markers}

Preliminary neuropsychological test data indicated that prodromal patients who subjectively experience information-processing deficits may also be characterised by objectively measurable disturbances. Particularly, attention, memory systems of different modalities and verbal capacities seem to be affected; however, these abnormalities were significantly less severe than in patients who had already developed schizophrenia.

Impaired attention was described as one of the strongest candidate markers of a susceptibility to schizophrenia in highrisk research (Cornblatt \& Obuchowski, 1997). Although other neurocognitive markers have not been studied prospectively in larger samples and their specificity for schizophrenia remains to be established, there is evidence from first-episode patients that memory and executive functions are further candidates of profound impairments (e.g. Saykin et al, 1994; Hutton et al, 1998). Memory performance in schizophrenia has been explained by primary semantic and episodic memory deficits reflecting a dysfunction of temporohippocampal subsystems (e.g. Tamlyn et al, 1992) and by less efficient executive functions, such as encoding and search strategies involving primarily prefrontal regions. The latter interpretation is consistent with the finding in this and many other studies (Aleman et al, 1999) that recognition performance is better than free recall memory performance, which remained stable even when both memory tasks were matched for psychometric difficulty (Calev, 1984). The predominant role of the prefrontal cortex for memory performance is also consistent with MRI studies showing correlations between memory dysfunctions and the volume of prefrontal rather than temporal regions (Seidman et al, 1994). Furthermore, the significant impairment in visual memory suggests that memory dysfunction is not restricted to left hemispheric processing of information. Finally, cognitive functions associated with dorsolateral prefrontal cortex such as planning, initiating and monitoring search and retrieval processes in long-term memory systems are particularly important for verbal fluency tasks, which proved to be the most sensitive neuropsychological indicator of performance deficits in the present data. Some of the observed neurocognitive dysfunctions in prodromal patients might be because of the low performance in verbal IQ. However, verbal IQ was correlated significantly with verbal fluency, indicating that both tests measure similar functions.

\section{Future directions}

In summary, prodromal patients who were selected on the basis of self-perceived disturbances, i.e. basic symptoms, also

Table 4 Differences in neurocognitive functions between prodromal patients (P), patients with schizophrenia (S), and normal controls (C); Mann-Whitney U-tests (mean (s.d.))

\begin{tabular}{|c|c|c|c|c|c|}
\hline Neurocognitive function & Controls, $n=29$ & Prodrome, $n=29$ & Schizophrenia, $n=29$ & $P$ for $P$ v. $C$ & $P$ for $P v . S$ \\
\hline Visual backward masking (\% hits) & $86.93(5.82)$ & $84.71(14.04)$ & $75.19(14.72)$ & 0.680 & $<0.001$ \\
\hline Attention (\% hits) & $81.47(10.37)$ & $72.66(18.26)$ & $53.52(20.23)$ & 0.044 & 0.001 \\
\hline Spatial working memory (pixel distance to target) & $55.73(11.54)$ & $55.79(18.24)$ & $86.51(39.96)$ & 0.529 & $<0.001$ \\
\hline \multicolumn{6}{|l|}{ Verbal memory (no. of words) } \\
\hline Free recall (trials I-5) & $12.11 \quad(1.50)$ & $11.21 \quad(1.69)$ & $9.46(2.4 I)$ & 0.044 & 0.004 \\
\hline Recognition & $14.14(1.36)$ & $13.97(1.45)$ & $12.00(2.82)$ & 0.426 & 0.006 \\
\hline Verbal fluency (no. of words) & $21.64(4.74)$ & $17.57(4.14)$ & $14.75(5.44)$ & 0.002 & 0.039 \\
\hline Visual memory (copy minus delay; standard score 0-36) & $8.85(5.13)$ & $12.36(5.51)$ & 17.48 (7.32) & 0.014 & 0.006 \\
\hline Wisconsin Card Sorting Test (\% perseverative errors) & $10.78(5.95)$ & $11.36(5.16)$ & I5.21 (7.52) & 0.380 & 0.023 \\
\hline
\end{tabular}


show objectively measurable neurocognitive deficits. The overall impairment profile of these patients is in accordance with models not focusing on single brain areas, but describing the disruption of fundamental neural circuits encompassing cortical-cerebellar-thalamic-cortical subsystems (e.g. Andreasen et al, 1998). The interrelation of self-perceived and objectively measured neuropsychological deviances in 'at-risk states' and their contributions to the prediction of psychosis merit further investigation. Within the German Research Network on Schizophrenia a set of multi-centre studies now explore the field of early detection and of early intervention more systematically, including prediction studies with psychopathological, neurophysiological, brain imaging and neuropsychological parameters.

\section{REFERENCES}

Aleman, A., Hijman, R., de Haan, E. H., et al (1999) Memory impairment in schizophrenia: a meta-analysis. American Journal of Psychiatry, 156, 1358-1366.

American Psychiatric Association (1987) Diagnostic and Statistical Manual of Mental Disorders (3rd edn, revised) (DSM-III-R). Washington, DC: APA.

- (1994) Diagnostic and Statistical Manual for Mental Disorders (4th edn) (DSM-IV). Washington, DC: APA

\section{Andreasen, N. C., Paradiso, S. \& O'Leary, D. S.}

(1998) 'Cognitive dysmetria' as an integrative theory in schizophrenia: a dysfunction in cortical-subcorticalcerebellar circuitry? Schizophrenia Bulletin, 26, 203-218.

Calev, A. (1984) Recall and recognition in chronic nondemented schizophrenics: Use of matched tasks. Journal of Abnormal Psychology, 93, 172-177.

Chapman, L. J., Chapman, J. P. \& Raulin, M. L. (1976) Scales for physical and social anhedonia. Journal of Abnormal Psychology, 85, 374-382.

Cornblatt, B. \& Obuchowski, M. (1997) Update of high-risk research. International Review of Psychiatry, $\mathbf{9}$ 437-447.

_ , Lenzenweger, M. F. \& Erlenmeyer-Kimling, L. (1989) The Continuous Performance Test, Identical pairs version: II. Contrasting attentional profiles in schizophrenic and depressed patients. Psychiatry Research, 9, 65-85.

_ , Obuchowski, M., Schnur, D., et al (1998) Hillside study of risk and early detection in schizophrenia. British journal of Psychiatry, 172, (suppl. 33), 26-32.

Gross, G. (1989) The 'basic' symptoms of schizophrenia British Journal of Psychiatry, 155 (suppl. 7), 21-25.

—, Huber, G., Klosterkötter, J., et al (1987) Bonn Scale for the Assessment of Basic Symptoms - BSABS. Berlin: Springer.

Häfner, H., Riecher-Rössler, A., Hambrecht, M., et al (1992) IRAOS: An instrument for the assessment of the onset and early course of schizophrenia. Schizophrenia Research, 6, 209-223.

_, Maurer, K., Löffler, W., et al (1993) The influence of age and sex on the onset and early course of schizophrenia. British Journal of Psychiatry, 162, 80-86.

\section{CLINICAL IMPLICATIONS}

Self-perceived disturbances of cognition and perception may indicate an increased risk for psychosis.

- Persons defined as 'prodromal' on the basis of psychopathology hold an intermediate position between controls and patients with schizophrenia on a number of neuropsychological measures.

- Information processing, both subjective and objective, is a promising level of assessment in the prediction of psychosis.

\section{LIMITATIONS}

The presented data are mostly cross-sectional and the (short-term) follow-up sample is small. This may limit statistical power.

- The paper focuses on neuropsychological disturbances, although a combination of different areas of assessment is needed to improve prediction.

Treatment was not monitored systematically and its preventive effect could not be taken into account.

MARTIN HAMBRECHT, MD, MICHAEL LAMMERTINK, MD, JOACHIM KLOSTERKÖTTER, MD,

EVELINE MATUSCHEK, MA, RALF PUKROP, PhD, Department of Psychiatry and Psychotherapy, University of Cologne, Germany

Correspondence: Professor Martin Hambrecht, Klinik für Psychiatrie und Psychotherapie, Evang. Krankenhaus Elisabethenstift, Landgraf-Georg-Str. 100, 64287 Darmstadt, Germany. E-mail:

hambrecht.martin@krankenhaus-elisabethenstift.de

_ , Nowotny, B., Löffler, W., et al (1995) When and how does schizophrenia produce social deficits? European Archives of Psychiatry and Clinical Neuroscience, 246, 17-28.

_ , Löffler, W., Maurer, K., et al (1999) Depression, negative symptoms, social stagnation and social decline in the early course of schizophrenia. Acta Psychiatrica Scandinavica, 100, 105-118.

Heaton, R. K., Chelune, G. J., Talley, J. L., et al (1993) Wisconsin Card Sorting Test Manual. Odessa, FL: Psychological Assessment Resources.

\section{Huber, G., Gross, G. \& Schüttler, R. (1979)} Schizophrenie Verlaufs- und sozialpsychiatrisch Langzeituntersuchungen an den 1945-1959 in Bonn hospitalisierten schizophrenen Kranken. Berlin: Springer.

Hutton, S. B., Puri, B. K., Duncan, L. J., et al (1998) Executive function in first-episode schizophrenia. Psychological Medicine, 28, 463-473.

Jackson, H. J., McGorry, P. D. \& Dudgeon, P. (1995) Prodromal symptoms of schizophrenia in first-episode psychosis: Prevalence and specificity. Comprehensive Psychiatry, 36, 24I-250.

Jaeschke, R., Guyatt, G. H. \& Sackett, D. L. (1994) User's guide to the medical literature. III. How to use an article about a diagnostic test. B.What are the results and will they help me in caring for my patients? Journal of the American Medical Association, 27I, 703-707.
Kay, S. (1991) Positive and Negative Syndromes in Schizophrenia. Assessment and Research. New York: Brunner-Mazel.

Klosterkötter, J., Hellmich, M., Steinmeyer, E. M., et al (200I) Diagnosing schizophrenia in the initial prodromal phase. Archives of General Psychiatry, $\mathbf{5 8}$ 158-164.

Larsen, T. K., Johannessen, J. O. \& Opjordsmoen, S. (1998) First-episode schizophrenia with long duration of untreated psychosis. British Journal of Psychiatry, 172 (suppl. 33), 45-52.

Lawrie, S. M. \& Abukmeil, S. S. (1998) Brain abnormality in schizophrenia. A systematic and quantitative review of volumetric magnetic resonance imaging studies. British Journal of Psychiatry, 172, 110-120.

Lehrl, S. (1995) Mehrfachwahl-WortschatzIntelligenztest: MWT-B. Balingen: Perimed-spitta.

Lezak, M. D. (1983) Neuropsychological Assessment. New York: Oxford University Press.

Loebel, A. D., Lieberman, J. A., Alvir, J. M. J., et al (1992) Duration of psychosis and outcome in first-episode schizophrenia. American Journal of Psychiatry, 149, 1183-1188.

McGlashan, T. H. (1999) Duration of untreated psychosis in first-episode schizophrenia: marker or determinant of course? Biological Psychiatry, 46, 899-907. 
McGorry, P. D., Edwards, J., Mihalopoulos, S. M. (1996) EPPIC: An evolving system of early detection and optimal management. Schizophrenia Bulletin, 22. 305-326.

_ , Adlard, S., Yung, A., et al (1999) Detection and intervention in pre-psychotic schizophrenia (abstract). Current Opinion in Psychiatry, 12 (suppl. I), 63.

_, McKenzie, D., Jackson, H. J., et al (2000) Can we improve the diagnostic efficiency and predictive power of prodromal symptoms of schizophrenia? Schizophrenia Research, 42, 91-100.

Mohamed, S., Paulsen, J. S., O'Leary, D., et al (1999) Generalized cognitive deficits in schizophrenia: a study of first-episode patients. Archives of General Psychiatry, 56, 749-754

Mrazek, P. J. \& Haggerty, R. J. (eds) (1994) Reducing Risks for Mental Disorders: Frontiers for Preventive
Intervention Research. Washington, DC: National Academy Press.

Rey, A. (1964) L'Examen Clinique en Psychologie. Paris: Presses Universitaires de France.

Saykin, A. J., Shtasel, D. L., Gur, R. E, et al (1994) Neuropsychological deficits in neuroleptic naive patient with first-episode schizophrenia. Archives of General Psychiatry, 5I, I24-I3I.

Scherbarth-Roschmann, P. \& Hautzinger, M. (1991) Zur psychometrischen Erfassung von Schizotypie. Methodische Überprüfung und erste Validierugn von zwei Skalen zur Erfassung von Risikomerkmalen. Zeitschrift für Klinische Psychologie, 20, 238-250.

Seidman, L. J., Yurgelon-Todd, D., Kremen, W. S., et (1994) Relationship of prefrontal and temporal lobe MR measures to neuropsychological performance in chronic schizophrenia. Biological Psychiatry, 35, 235-246.
Spitzer, M. (1993) The psychopathology, neuropsychology, and neurobiology of associative and working memory in schizophrenia. European Archives of Psychiatry and Clinical Neuroscience, 243, 57-70.

Tamlyn, D., McKenna, P. J., Mortimer, A. M., et al (1992) Memory impairment in schizophrenia: its extent, affiliations and neuropsychological character. Psychological Medicine, 22, I0I-115.

Wittchen, H. U. \& Fydrich, T. (1997) Strukturiertes Klinisches Interview für DSM-IV. Manual zum SKID-I und SKID-II. Göttingen: Hogrefe.

World Health Organization (1992) Tenth Revision of the International Classification of Diseases and Related Disorders (ICD-10). Geneva: WHO.

Yung, A. R., Phillips, L. J., McGorry, P. D., et al (1998) Prediction of psychosis. A step towards indicated prevention of schizophrenia. British Journal of Psychiatry, I72 (suppl. 33), 14-20. 